\title{
Chemical similarities between the Galactic bulge and local thick disk red giant stars: analysis from optical data
}

\author{
Alan Alves-Brito ${ }^{1}$, Jorge Meléndez ${ }^{2}$, and Martin Asplund ${ }^{3}$ \\ ${ }^{1}$ Universidade de São Paulo, IAG \\ email: abrito@astro.iag.usp.br \\ ${ }^{2}$ Centro de Astrofísica da Universidade do Porto, Portugal \\ ${ }^{3}$ Max Planck Institut für Astrophysik, Germany
}

\begin{abstract}
The Galactic structure and composition remain as one of the greatest open problems in modern astrophysics. We show here that there are chemical similarities between the Galactic bulge and local thick disk red giant stars. This finding puts strong constraints on the IMF, SFR and chemical enrichment timescale of the bulge and thick disk. Our results are based upon a detailed elemental abundance analysis of 80 high $\mathrm{S} / \mathrm{N}$ and high resolution optical spectra of giant stars, in the range $-1.5<[\mathrm{Fe} / \mathrm{H}]<+0.5$.
\end{abstract}

Keywords. stars: abundances, Galaxy: bulge, Galaxy: disk

\section{Introduction}

Our own Galaxy offers a remarkable opportunity for testing different scenarios of galaxy formation and assembly. In this sense, the bulge and disk play a crucial role because these two Galactic subcomponents mostly contribute to the Galaxy total stellar mass (e.g. Wyse 2009). Furthermore, the bulk of bulge and thick disk stars appear to be old (Ortolani et al. 1995; Zoccali et al. 2003; Bensby et al. 2007).

On one hand, the bulge's old ages and enhancement of $\alpha$-elements - as revealed by the detailed chemical analysis of giant stars in the field (e.g. McWilliam \& Rich 1994; Cunha \& Smith 2006; Zoccali et al. 2006; Lecureur et al. 2007; Fulbright et al. 2007; Meléndez et al. 2008; Ryde et al. 2009a,b) and in globular clusters (e.g. Meléndez et al. 2003; Alves-Brito et al. 2006; Barbuy et al. 2008) -, suggest that the Galactic bulge must have formed rapidly during intensive star formation (the "classical" scenario). On the other hand, the Galactic bulge's boxy shape is also morphologically consistent with a "pseudo-bulge" scenario, which indicates the bulge must have formed as a consequence of secular evolution through dynamical instability of an already established inner disk (see Kormendy \& Kennicutt 2004, for a review).

Meléndez et al. (2008) analysed high resolution infrared spectra of both bulge and thick disk giants with similar stellar parameters and found that the bulge is in fact chemically very similar to the thick disk in $[(\mathrm{C}, \mathrm{N}, \mathrm{O}) / \mathrm{Fe}]$. Here we present the results based on oxygen and other $\alpha$-elements $(\mathrm{Mg}, \mathrm{Si}$, Ti and $\mathrm{Ca})$ as derived from high resolution optical data.

\section{Results and conclusions}

Figure 1 shows the pattern of $[\alpha / \mathrm{Fe}]$ versus $[\mathrm{Fe} / \mathrm{H}]$ for the bulge and thick disk stars. Non-weighted least squares fit to $[\alpha / \mathrm{Fe}]-[\mathrm{Fe} / \mathrm{H}]$ for metal-poor $([\mathrm{Fe} / \mathrm{H}]<-0.5)$ and 


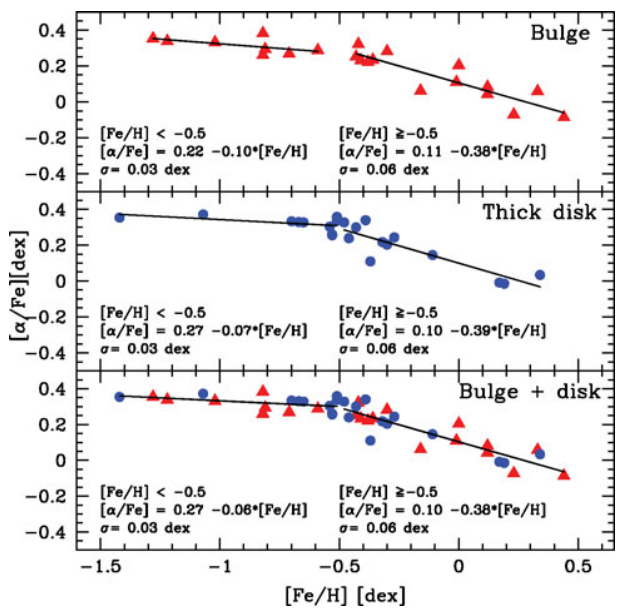

Figure 1. Mean $[\alpha / \mathrm{Fe}]$ vs. $[\mathrm{Fe} / \mathrm{H}]$ for the sample. Refer to the text for more details.

metal-rich $([\mathrm{Fe} / \mathrm{H}] \geqslant-0.5)$ bulge $(t o p)$, thick disk (middle) and both bulge and thick disk (bottom) stars is also displayed. As one can see, similar relations can fit both stellar populations, with a scatter as low as $\sigma=0.03$ dex. This finding suggests that the bulge and local thick disk giants have essentially identical chemical abundance ratios. Thus, the bulge and local thick disk stars experienced similar formation timescales, star formation rates and initial mass functions. Our results and conclusions are discussed in detail in Alves-Brito et al. (2009, A\&A, submitted).

Interestingly, Bensby et al. (2009) have found that microlensed dwarfs in the Galactic bulge present $[\alpha / \mathrm{Fe}]$ abundance ratios similar to those of dwarfs in the Galactic thick disk, which agrees with our results for giant stars.

\section{Acknowledgements}

AAB acknowledges financial support from CAPES (4685-06-7) and FAPESP (04/ 00287-9).

\section{References}

Alves-Brito, A., Barbuy, B., Zoccali, M., et al. 2006, A\&SA, 460, 269

Barbuy, B., Alves-Brito, A., Ortolani, S., et al. 2008, PhST, 133a, 4032

Bensby, T., Zenn, A. R., Oey, M. S., \& Feltzing, S. 2007, ApJ, 663, 13

Bensby, T., Feltzing, S., Johnson, J. A., et al. 2009, in press, arXiv0908.2779

Cunha, K. \& Smith, V. V. 2006, ApJ, 651, 491

Fulbright, J. P., McWilliam, A., \& Rich, R. M. 2007, ApJ, 661, 1152

Kormendy, J. \& Kennicutt, Jr., R. C. 2004, ARA\&A, 42, 603

Lecureur, A., Zoccali, M., Hill, V., et al. 2007, A\&A, 465, 799L

McWilliam, A. \& Rich, R. M. 1994, ApJS, 91, 749

Meléndez, J., Barbuy, B., Bica, E., et al. 2003, A\& A, 411, 417

Meléndez, J., Asplund, M., Alves-Brito, A., et al. 2008, A\&A, 484, L21

Ortolani, S., Renzini, A., Gilmozzi, R., et al. 1995, Nature, 377, 701

Ryde, N., Edvardsson, B., Gustafsson, B., et al. 2009a, A\&SA, 496, 701

Ryde, N., Edvardsson, B., Gustafsson, B., et al. 2009b, A\&A, in press, arXiv:0910.0448

Wyse, R. 2009, IAUS, 258, 11

Zoccali, M., Renzini, A., Ortolani, S., et al. 2003, A\&A, 399, 931

Zoccali, M., Lecureur, A., Barbuy, B., et al. 2006, A\&A, 457, 1 